\title{
Financial interbanking networks resilience under shocks propagation
}

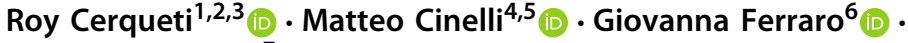 \\ Antonio lovanella ${ }^{7}$
}

Accepted: 21 January 2022

๑) The Author(s), under exclusive licence to Springer Science+Business Media, LLC, part of Springer Nature 2022

\begin{abstract}
The concept of resilience-i.e., the ability of a unified structure to absorb shocks-is of high relevance in the context of network modelling and analysis, mainly when referred to finance. This paper starts from this premise, and deals with the resilience of a financial interbanking system. At this aim, we firstly introduce a new measure of the resilience of a network, by taking into full consideration the influence of the topology of the network and the weights of its links in the shocks propagation; then, we build one financial network model related to the quarterly-based interbanking sector, whose weights are calibrated on high quality empirical data; lastly, we compute the resilience measure of the considered networks. A discussion of the results is provided, by considering both finance and network theory perspectives.
\end{abstract}

Keywords Financial interbanking networks $\cdot$ Network modelling and analysis $\cdot$ Resilience Shock propagation · Interconnectedness · Bank for International Settlements

$\triangle$ Antonio Iovanella

antonio.iovanella@unint.eu

Roy Cerqueti

roy.cerqueti@uniroma1.it

Matteo Cinelli

matteo.cinelli@uniroma1.it

Giovanna Ferraro

giovanna.ferraro@unipr.it

1 Department of Social and Economic Sciences, Sapienza University of Rome, P.le A. Moro, 5, 00185 Rome, Italy

2 GRANEM - Université d'Angers, Angers, France

3 School of Business, London South Bank University, UK

4 Department of Computer Science, Sapienza University of Rome, Viale Regina Elena, 295, 00161 Rome, Italy

5 Italian National Research Council — Institute for Complex Systems, Via dei Taurini 19, 00185 Rome, Italy

6 Department of Engineering and Architecture, University of Parma, Parco Area delle Scienze, 181/A, 43124 Parma, Italy

7 School of Economics, Università degli Studi Internazionali di Roma - UNINT, Via Cristoforo Colombo, 200, 00147 Rome, Italy 


\section{Introduction}

Assessing the ability of a system to absorb shocks is a very relevant task. Indeed, it is not rare to assist at macroscopic fluctuations of a complex system generated by a microscopic impulse occurring in one of its components. The consequences of such sources of instability might be devastating; hence, their prevention and management represent targets for all the actors operating around the observed system.

From this perspective, the high attention paid to the resilience is considered useful in order to acquire insights concerning complex systems and, in particular, complex networks. Starting from the seminal paper of Albert et al. (2000), resilience in complex networks was investigated theoretically (Gao et al. 2016) and then extended by considering the effects of different node removal strategies on the network structure (Ferrraro and Iovanella 2018; Iyer et al. 2013) or as a function of nodes' mixing preferences (D’Agostino et al. 2012).

For the special theme of the resilience, it is natural to capture the propagation of a shock over a system as a transition from different sites through their connecting links. In the scientific literature, the study of the resilience is mainly related to the analysis of a performance measure, typically the diameter of the network, under two different schemes of stress depending on a targeted attack to a particular node or considering the random failure of a node, viewed as a network error (Albert et al. 2000; Chen et al. 2015). Such analysis are relatively easy to perform and this explains why the models of resilience of a system are framed in the context of complex networks (Ferrraro and Iovanella 2018).

In this paper, the assessment of the resilience is performed by taking into considerations a new measure based on the shocks occurring in one of the nodes and on its propagation over the links of the network. We assume that the propagation of a shock from a node to another one occurs over the shortest path connecting the nodes. In so doing, we develop a model where the nodes are immunized from the subsequent effects of the shock, once they are infected for the first time by its propagation. This condition is particularly reasonable in several realworld situations. Moreover, it offers also relevant advantages under the perspective of the computational complexity of the applications of such a resilience measure. Indeed, under a purely methodological point of view, the considered resilience measure is similar to that presented in Cerqueti et al. (2019), by overcoming its limitations in terms of its applicability opportunities when focusing on the shortest paths. Indeed, as indicated in Cinelli et al. (2017), the computation time of all simple paths grows exponentially with the size of the network (Fortune et al. 1980), thus only small networks can be analysed in an acceptable time. Importantly, in line with (Cerqueti et al. 2019), we assume that the propagation of the shock is opportunely rescaled as the distance from the shocked nodes increases. In doing so, we provide a versatile device which includes either amplified or dampened effects of the distance from the shocked node-where the nature of the effects has to be identified on the basis of the considered real world case. In this context, a relevant role is played by the identification of the size of the shock (on this, see also Acemoglu et al. 2015).

The proposed resilience measure shows some clear advantages with respect to the existing ones. Indeed, we point out that the most popular resilience measure in the environment of complex networks is based on the nodes removal, with the assessment of the reaction of the overall system to such an impulsive shock (see Albert et al. 2000). Under a different perspective, we can also consider resilience measures based on a cascade model (see e.g. Rosas-Casals et al. 2015; Scala et al. 2016). In such a context, the failure of a node generates an avalanche-like event that may generate the disruption of the entire system. Such measures are not suitable to provide a proper modelling of our framework. Indeed, a resilience measure 
based on nodes removal is not a reliable device in a context such as the financial one-where the actors go in distress but are not removed from the system; furthermore, cascade-based models are associated to collapsing systems, while here we discuss the direct reaction of the single nodes to the shocks propagating from other nodes. In this respect, the resilience measure proposed in our paper is suitable for financial applications, in that it includes also the propagation of the financial distress from a country to another one.

As we will see, the methodological proposal is tested over a relevant financial network associated to the interbanking sector. The motivations for this choice lies in the paradigmatic relevance of the financial context, mainly when one deals with the concept of resilience. In fact, accounting for shocks propagation in finance contributes to describe a pivotal feature of financial systems, which has been tested by several paradigmatic cases such as the financial contagion associated to the Lehman Brothers' bankruptcy (Dumontaux and Pop 2013). This explains why in the wide world of complex networks, those of financial nature are of particular interest (Allen and Babus 2008). Many applications were studied considering dynamic evolution (Peron et al. 2012), incomplete information (Cinelli et al. 2021), risk management (Nagurney and Ke 2006) and diffusion of contagion and its relationship with the network structure (Elliott et al. 2014,Glasserman and Peyton Young 2016). Indeed, the constitutive property of a network model-i.e., the presence of disaggregated units which are mutually interconnected-is crucial when the aim is to describe financial patterns (see e.g. Nedovic and Devedzic 2002 or the review in Rada (2008)).

In detail, we propose a complex network model for the interbanking sector, where nodes are countries and links are weighted on the basis of the mutual exposures among the banks headquartered in the countries. Specifically, we hypothesize that the stronger the mutual exposures among the banks, the higher the weights of the links among the related countries, as intuitively should be.

Following previous contributions in the quantitative finance literature, we have retrieved the empirical quarterly data from the Bank for International Settlements (BIS) database (see e.g. Bongini et al. 2018; Cerqueti et al. 2020; Cinelli et al. 2021; Demir and Onder 2019; Giudici and Spelta 2016; Minoiu and Reyes 2013; Minoiu et al. 2015). The BIS is owned by 62 central banks covering about 95\% of the world's GDP (BIS database www.bis.org). The analysis is carried out also at a temporal level. The period under investigation is 2005-2020 (this latter limited to the first two quarters); such a multiperiodal framework leads to a deep analysis of the resilience evolution in the considered network, in that we build a network for each quarter of the investigated period. Moreover, the financial crisis due to Lehman Brothers' failure (Dumontaux and Pop 2013) is also taken into consideration.

It is also important to notice that BIS networks are not small enough to face the computational complexity associated to the computation of the resilience measure when not restricting to the shortest paths. However, we point out that such an approximation is quite reasonable in our specific context, since it describes the real situation of a financial contagion walking over the easiest and most direct propagation patterns [see the discussion on this point in the monograph (Chevallier et al. 2019)]. In this respect, our methodological proposal is also able to model the real world situation where a node is infected only once-hence, through the shortest paths-and does not react to subsequent solicitations stemming from the same shocks.

Results on the BIS networks offer a complete view of the considered interbanking sector over the period under investigation. In particular, we observe different resilience patterns as time goes from 2005 to 2020 which significantly correlate with the recorded financial history of such years. 
Interestingly, the action of the amplifying/dampening effect of the shocks as the distance from the shocked node increases is relevant. Moreover, there is a reasonable dependence of the resilience of the network on the size of the shock. The obtained outcomes state clearly that large interbank exposures lead to a high level of resilience. In this respect, the years related to the recent economic distresses - the Lehman Brothers' bankruptcy in 2008 and the COVID-19 pandemic in 2020 - are associated to resilience peaks, in agreement with the stylized fact that correlation among banks increases in situations of financial distress.

The paper is organised as follows. Section 2 introduces the resilience measure used in our analysis, by including also a discussion related to the complexity of the considered tool. Section 3 outlines the proposed interbanking network model. Section 4 reports the results of the simulations performed and the related discussions. Finally, Sect. 5 offers some conclusive remarks and outlines directions for future research.

\section{The resilience measure}

Herein, we introduce the measure of resilience that we will use in the analyses as well as the rationale behind the construction of such a measure. At this aim, as we will see in detail below, we start from Cerqueti et al. (2019) and remove the limitations of the resilience measure in the quoted paper related on its computational complexity — hence, preventing to its applicability in contexts of highly dense networks.

We consider a set $V=\{1, \ldots, n\}$, representing the set of the nodes of a complex directed and weighted network.

The adjacency matrix of the network is denoted by $\mathbf{W}=\left(w_{i j}\right)_{i, j \in V}$. Matrix $\mathbf{W}$ is not necessarily symmetric.

We assume that $w_{i j}>0$ if and only if there exists a directed link between nodes $i$ and $j$. In doing so, the adjacency matrix $\mathbf{W}$ provides a straightforward identification of the links connecting the nodes of the network. When needed, we refer to $E$ as the set of the links of the network.

We now give an integer $k \geq 2$ and introduce the $k$-paths with starting node $i_{0} \in V$ by $p_{i_{0}}^{(k)}=\left\{i_{0} \rightarrow i_{1} \rightarrow \cdots \rightarrow i_{k-1} \rightarrow i_{k}\right\}$, with $i_{0}, i_{1}, \ldots, i_{k-1}, i_{k}$ distinct nodes in $V$ and $i_{h-1} \rightarrow i_{h} \in E$, for each $h=1, \ldots, k$. The node $i_{k}$ is said to be the terminal node of $p_{i_{0}}^{(k)}$. When needed, we will refer to $p_{i_{0}, i_{k}}^{(k)}$ to specify both the initial node and the terminal node.

Intuitively, the (aggregate) weight of a generic $k$-path starting from $i_{0}$ and with terminal node $i_{k}$ is

$$
w\left(p_{i_{0}, i_{k}}^{(k)}\right)=\sum_{h=1}^{k} w_{i_{h-1} i_{h}} .
$$

We assume that the diameter of the network is $\bar{k}$, so that $k=2, \ldots, \bar{k}$.

All the $k$-paths of the network are collected in

$$
\mathcal{P}^{(k)}=\bigcup_{i_{0} \in V} \mathcal{P}^{(k)}\left(i_{0}\right),
$$

where $\mathcal{P}^{(k)}\left(i_{0}\right)$ is the collection of the $k$-paths whose starting node is $i_{0}$.

The set $\mathcal{P}^{(k)}$ in (2) can be quite large, mainly when the considered network is dense and it has a large number of nodes. Indeed, given two nodes $i_{0}, i_{k} \in V$, one can have a large number of different paths starting from $i_{0}$ and with terminal node $i_{k}$. Such an evidence is 
the ground of the computational complexity arising in the resilience measure proposed by Cerqueti et al. (2019).

For this reason, we conveniently restrict our attention to a special subset of $\mathcal{P}^{(k)}$.

At this aim, given two nodes $i_{0}, i_{k} \in V$, we introduce the shortest path with starting point $i_{0}$ and terminal point $i_{k}$ as

$$
p_{i_{0}, i_{k}}^{\min }=\operatorname{argmin}\left\{w\left(p_{i_{0}, i_{k}}\right): p_{i_{0}, i_{k}} \in \bigcup_{h=1}^{\bar{k}} \mathcal{P}^{(k)}\right\} .
$$

Evidently, formula (3) entails that the shortest path with initial value $i_{0}$ and terminal value $i_{k}$ is not necessarily unique. Therefore, once $i_{0}$ and $i_{k}$ are fixed, being a shortest path connecting $i_{0}$ with $i_{k}$ is an equivalence relation. Thus, we can identify the equivalence class collecting the shortest paths from $i_{0}$ to $i_{k}$, namely $\left[p_{i_{0}, i_{k}}^{\text {min }}\right]$. Hereafter, we will consider only one element of each equivalence class $\left[p_{i_{0}, i_{k}}^{m i n}\right]$, without duplicating the shortest paths associated to the same couple of nodes. We denote the representative element of $\left[p_{i_{0}, i_{k}}^{\text {min }}\right]$ as $p_{i_{0}, i_{k}}^{\text {min }}$, and we refer to it simply as shortest path from $i_{0}$ to $i_{k}$. All the shortest paths of the network - clearly, to be intended in the sense of the representative elements-are collected in a set $\mathcal{P}_{\text {short }}$.

We are now in the position of defining the shocks, which are here local events occurring to the nodes of the network. The entity of the shock plays a key role in the analysis of the resilience of the network. Such an entity is assumed to be captured by a positive number $\xi \in(0,+\infty)$, and it grows-i.e., the effect of the shock becomes stronger-as the value of $\xi$ increases.

The shock starts from a given node $i_{0} \in V$ and propagates over the shortest paths with initial value $i_{0}$. More specifically, we do not prevent the shock to propagate over all the $k$ paths, but we do not consider the effects of the shock propagating outside the shortest paths. Practically, this means that the effect of the shock on a node connected to $i_{0}$ is registered only at the first time in which such a node is reached-thus, through the shortest path. After that, the node is infected, and it does not react to subsequent solicitations stemming from the same shock. The way propagation occurs depends on the weights of the shortest $k$-paths, on the entity of the shock and on the distance from the node subject to the shock.

According to Cerqueti et al. (2019), we consider the propagation on a generic $k$-path $p_{i_{0}}^{(k)}$ with weight as in (1) and define a discount factor $\delta \in[0,+\infty)$, that captures the effect of the distance on the propagation as follows

$$
\xi_{h}=\xi \sum_{s=1}^{h} w_{i_{s-1} i_{s}} \delta^{h-s+1},
$$

where $\xi_{h}$ is the entity of the shock at node $i_{h}$, for each $h=0, \ldots, k$ and $\xi_{0}=\xi$.

We also include a propagation condition, which is the core of the resilience measure. Indeed, if the shock is too weak, then the propagation motion stops. To formalize the propagation condition, we introduce a vector $\Gamma=\left(\gamma_{1}, \ldots \gamma_{\bar{k}}\right) \in(0,+\infty)^{\bar{k}}$ and assume that the existence of $s=0,1,2, \ldots, h-1$ such that $\xi_{s}<\gamma_{s}$ prevents the propagation of the shock to node $i_{h}$. The validity of the propagation conditions clusters the $k$-paths in two classes: the ones where the shock $\xi$ propagates and those where such a shock does not achieve the last node. The clusters are, of course, dependent also on the considered vector $\Gamma$. We denote by $\mathcal{P}_{\Gamma, \xi}^{(k)}$ the set collecting the $k$-paths - not necessarily the shortest $k$-paths-which satisfy the propagation condition for $\Gamma$ and $\xi$.

We are ready to define our concept of network resilience. 
Given the vector $\Gamma$ and the shock with entity $\xi$, we define the $\Gamma-\xi$-resilience measure of the network $N=(V, E)$ as

$$
\mu_{(\Gamma, \xi)}(N)=1-\sum_{k=1}^{\bar{k}} \theta_{k} \frac{\left|\mathcal{P}_{\Gamma, \xi}^{(k)} \cap \mathcal{P}_{\text {short }}\right|}{\left|\mathcal{P}^{(k)} \cap \mathcal{P}_{\text {short }}\right|},
$$

where $\theta_{1}, \ldots, \theta_{\bar{k}}$ are appropriately selected to be nonnegative weights such that $\sum_{k=1}^{\bar{k}} \theta_{k}=1$. We denote the vector of the weights by $\Theta=\left(\theta_{1}, \ldots, \theta_{\bar{k}}\right)$. The selection of $\Theta$ is implemented on the basis of the specific attention towards the different lengths of the paths when measuring the resilience of the network.

We observe that $\mu_{(\Gamma, \xi)}(N) \in[0,1]$ and it increases as the shocks propagate less easily over the network - so that, the network is more resilient. When $\mu_{(\Gamma, \xi)}(N)=1\left(\mu_{(\Gamma, \xi)}(N)=0\right.$, resp.), then the shock $\xi$ is absorbed (the shock $\xi$ propagates over all the paths, resp.).

\subsection{Complexity test}

The comparison between our proposal based on shortest paths and the approach of Cerqueti et al. (2019) — which takes into account all the paths-is associated to the discussion related to the complexity of the related algorithms. To face this relevant issue, we firstly provide a brief theoretical overview of the computational times in the two cases; then, we present an experiment supporting the theoretical side of the discussion.

The computation of all simple paths is well known to be a hard task (Fortune et al. 1980), while the set of the shortest paths is easy to compute since the time complexity is polynomial in the size $n$ of a graph $G$ (Ahuja et al. 1988).

Table 1 shows the comparisons between the computation of $\mu_{(\Gamma, \xi)}(N)$ when considering all the simple paths (as in Cerqueti et al. (2019)) and when computing only the shortest paths according to Eq. 5. The first column contains the type of network under examination, the second the cardinality of the set $\mathcal{P}^{(k)}$, the third the value for $\mu_{(\Gamma, \xi)}(N)$ and the fourth the computational time when computing all the simple paths. The following three columns show the same results when computing all the shortest paths and, finally, the last column reports the percentage deviation $\Delta_{\mu}$ between the two values of $\mu_{(\Gamma, \xi)}(N)$. The simulations have been performed on ten instances with $\xi=0.1$ and $\delta=1$. All the values in Table 1 are to be considered averaged over ten instances.

Since paths are the basic ingredient for our resilience measure, we followed the same general approach used in the analysis of shortest path problems (Demetrescu et al. 2009) and we tested the two versions of the measure on grid and random networks. For both we considered $n=\{25,50\}$ and $w_{i j}$ randomly chosen in the set $\{1,2, \ldots, 10\}$. Finally, in the case of random networks, we considered the values of density as $d=\{0.1,0.2\}$.

From Table 1 we may notice that in all the simulations, as expected, the computational times for $\mu_{(\Gamma, \xi)}(N)$ considering all shortest paths outperform the other case. Note also that the gaps in the resilience measures $\Delta_{\mu}$ are remarkably low (less than $1 \%$ ), i.e. we can use the shortest paths at the cost of a small difference in the resilience measure.

It is worth noting that the negligible difference between the case of all paths and that of shortest paths was quite expected. Indeed, by definition, the shocks propagate more likely over the shortest paths than in the non shortest cases. In the considered situation, the percentage of the paths that are of non shortest nature and allowing the propagation of the shock is so small that the deviation between the resilience measures introduced in (5) and that in Cerqueti et al. (2019) is not that relevant. 
Table 1 Result considering either the set of simple paths or the set of shortest paths in the $\mu_{(\Gamma, \xi)}(N)$ calculation for benchmark networks (averaged over 10 instances)

\begin{tabular}{|c|c|c|c|c|c|c|c|}
\hline \multirow[t]{2}{*}{ Network } & \multicolumn{3}{|c|}{ All simple paths } & \multicolumn{3}{|c|}{ All shortest paths } & \multirow[t]{2}{*}{$\Delta_{\mu}$} \\
\hline & $\left|\mathcal{P}^{(k)}\right|$ & $\mu_{(\Gamma, \xi)}(N)$ & Time & $\left|\mathcal{P}^{(k)}\right|$ & $\mu_{(\Gamma, \xi)}(N)$ & Time & \\
\hline Grid, $n=25$ & 185486.6 & 0.995 & $59.34 \mathrm{~m}$ & 639.6 & 0.987 & $4.21 \mathrm{~s}$ & $0.7 \%$ \\
\hline $\begin{array}{l}\text { Grid, } n=50 \\
\text { Random, }\end{array}$ & - & - & $>2 d$ & 2632.3 & 0.990 & $10.57 \mathrm{~s}$ & - \\
\hline $\begin{array}{l}n=25, d=0.1 \\
\text { Random, }\end{array}$ & 201.5 & 0.978 & $0.79 \mathrm{~s}$ & 137.9 & 0.976 & $0.41 \mathrm{~s}$ & $0.1 \%$ \\
\hline $\begin{array}{l}n=25, d=0.2 \\
\text { Random, }\end{array}$ & 84980,2 & 0.993 & $20.11 \mathrm{~m}$ & 532,3 & 0.984 & $2.03 \mathrm{~s}$ & $0.9 \%$ \\
\hline $\begin{array}{l}n=50, d=0.1 \\
\text { Random, }\end{array}$ & - & - & $>2 \mathrm{~d}$ & 2168.0 & 0.991 & $8.28 \mathrm{~s}$ & - \\
\hline$n=50, d=0.2$ & - & - & $>2 \mathrm{~d}$ & 2897.3 & 0.998 & $8.37 \mathrm{~s}$ & - \\
\hline
\end{tabular}

In the time columns, $\mathrm{s}$ are seconds, $\mathrm{m}$ are minutes, $\mathrm{h}$ are hours and $\mathrm{d}$ are days. In some rows results are not reported since the simulation ran out of memory after two days of computation

\section{The interbanking network model}

The financial context here considered is taken from the interbanking system and data are retrieved by the BIS web site database (BIS database www.bis.org). In particular, we refer to the activities associated to the consolidated banking statistics (CBS) and to the Locational Banking Statistics (LBS) of all the available countries (see the details in the Appendix).

The CBS captures the worldwide consolidated positions of internationally active banking groups headquartered in reporting countries. The CBS include the business of banks' foreign affiliates but exclude intragroup positions, similarly to the consolidation approach followed by banking supervisors.

The LBS provide information about the currency composition of banks'balance sheets and the geographical distribution of their counterparties. The LBS capture the outstanding financial assets and liabilities of internationally active banks located in reporting countries against counterparties residing in more than 200 countries. $^{1}$

The CBS and LBS data present different characteristics, even if they are associated to the same interbanking context. In our study, we consider the analysis conducted on LBS as a robustness check on the results obtained for CBS. We explain the reasoning behind this approach.

One of the reasons for preferring CBS is that consolidated statistics seems to be better suited for representing a risk-propagation scenario at aggregated level (Tonzer 2015). Moreover, taking into account the nationality of banks, rather than for instance their residency, could be deemed as a prudential way to involve countries in the process of risk propagation, especially considering the fact that BIS networks suffer of missing links by construction. Furthermore, consolidated data seem to be apt for research efforts that do not include an explicit geographical/spatial dimension as in our analysis.

1 Bank for International Settlements, Monetary and Economic Department, Reporting guidelines for the BIS international banking statistics, July 2019 (https://www.bis.org/statistics/bankstatsguide.pdf), accessed October $25^{t h}, 2021$. 
Nonetheless, some authors argues that LBS display some advantages with respect to CBS such as available cross-border bank capital flows data, exchange rate adjusted flow data and a longer time window (Minoiu et al. 2015; Minoiu and Reyes 2013; Tonzer 2015). For these reasons we repeat stress tests on the LBS dataset and compare it to the CBS dataset, in terms of our indicator $\mu$, in order to understand whether the resilience displayed by CBS data is affected by the consolidation.

The considered datasets deal with banking groups in terms of hosting or residence countries, so that banks are intuitively associated to countries. Therefore, for both CBS and LBS networks, we take countries as nodes in the set $V=\{1, \ldots, n\}$.

We assume that the weight $w_{i j}$ is based on the aggregated bank exposures $\alpha_{i j}$-expressed in billions of dollars - of the banking groups headquartered in country $i$ to the ones of country $j$. Specifically, we consider $w_{i j}=\alpha_{i j}$, for each $i, j \in V$.

We present quarterly data, ranging from the first quarter of 2005 to the fourth quarter of 2020. Thus, we have 64 networks - one network for each quarter. The number of nodes and links varies over the periods: for CBS network, nodes range from 202 to 215 (210.05 on average) and links from 1922 to 2882 (2448.78 on average); for LBS networks, nodes range from 209 to 213 (211.72 on average) and links from 2688 to 4942 (3864.72 on average).

For the sake of completeness, consider that countries in the dataset are divided in three different classes (Bongini et al. 2018): (i) the core, which considers the 26 reporting countries that have both incoming and outgoing exposure of international financial claims to the BIS (note that we report the actual number since countries have been added over the years); (ii) the 10 global systemically (GS) countries where at least one G-SIB bank is headquartered; ${ }^{2}$ (iii) the periphery, which considers those countries where only inflow is available. Figures 1 and 2 show the network of the fourth quarter of 2020 for CBS and LBS, respectively, while Figs. 3 and 4 illustrate the network of the GS elements in the same quarter for CBS and LBS, respectively.

Table 2 reported in Appendix shows the lists of core and GS countries. Such countries share a high number of links (see Figs. 1, 2, 3, and 4 in both of cases of CBS and LBS and for a temporal instance), meaning that-under a purely topological perspective, i.e. in the unweighted case - the corresponding network structure has a local density considerably higher than the rest of the network. For this reason, the topological structure of the BIS networks is considered to be of core-periphery type.

Interestingly, in the case of core-periphery strucures, network resilience has some peculiar characteristics that are worth mentioning (Cinelli et al. 2017). Indeed, in case of classical error and attack tolerance analysis, such networks are considered to be more resilient because of the presence of an high global cohesion, predominantly in the core. On the other hand, such networks are typically characterised by smaller values of the diameter, thus propagation towards other nodes is more likely to occur. Of course, the presence of the weights might lead to a departure of the weighted network from the topological core-periphery structure. However, the arguments above may be viewed as a support of the worthiness of our study.

In Fig. 5 we show the in-degree and the out-degree distributions for CBS and LBS networks, respectively, as yearly boxplots, throughout the observation period. Implicitly, such distributions provide a confirmation of the topological core-periphery structure. Indeed, despite the shapes of the two distributions the values of $y$-axis differ due to the fact thatas already mentioned above-the peripheral countries report only inflow. We observe, with reference to the out-degree, a high number of banks/countries that do not have exchanges.

2 G-SIB banks (Global Systemically Important Banks) are subject to more stringent requirements. See http:// www.fsb.org/2017/11/fsb-publishes-2017-g-sib-list/ for the updated list of banks and requirements. 


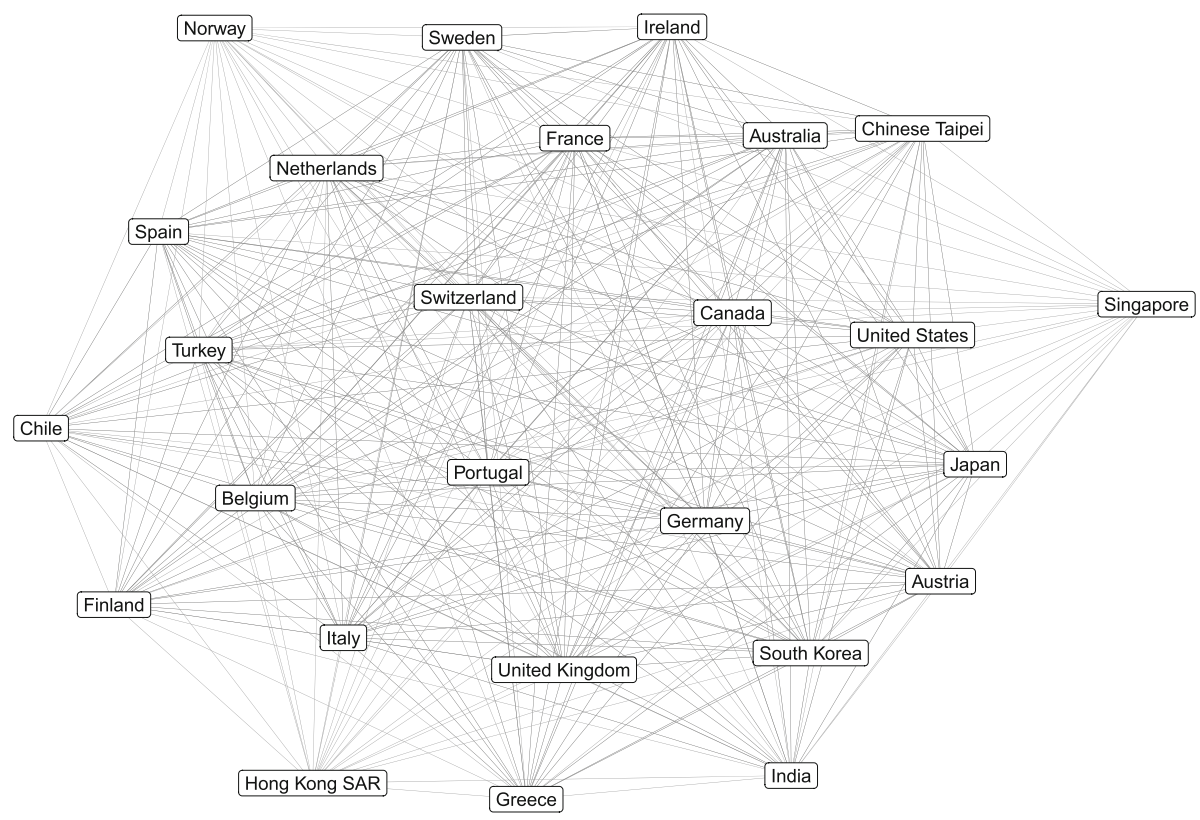

Fig. 1 Network of core elements (i.e. reporting countries) for the CBS fourth quarter of 2020. The existence of a link represents a positive cross-border exposure $\alpha_{i j}$ between the two nodes $i, j$

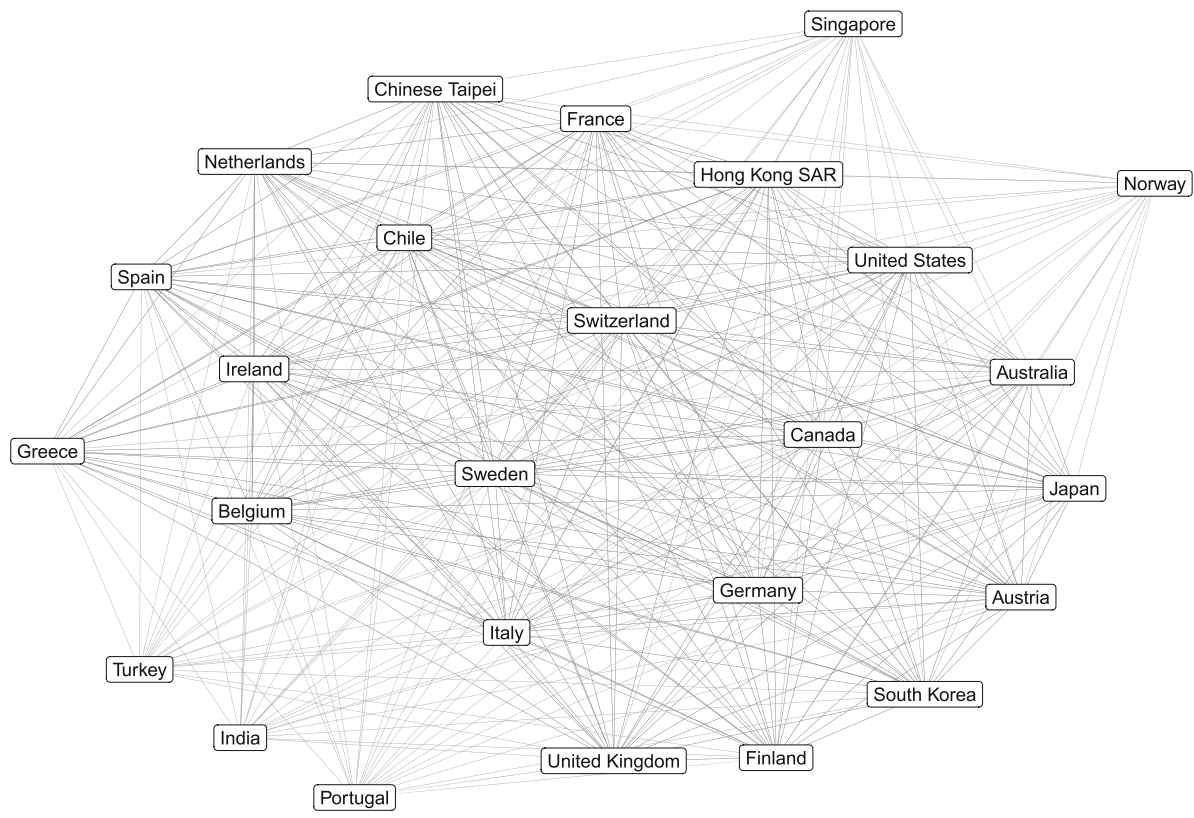

Fig. 2 Network of core elements (i.e. reporting countries) for the LBS fourth quarter of 2020. Also in this case, the existence of a link captures a positive cross-border exposure $\alpha_{i j}$ between the two nodes $i, j$ 


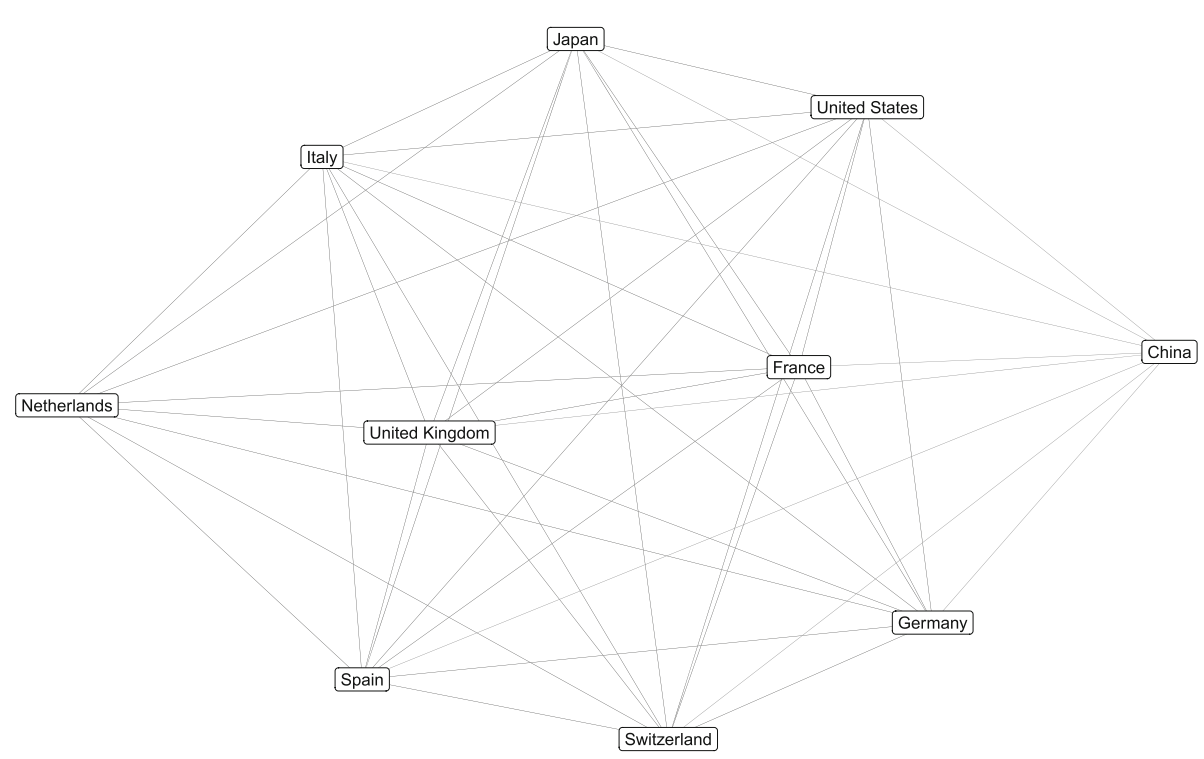

Fig. 3 Network of GS elements (i.e. countries where at least one global systemically important bank has its headquarter) for the CBS fourth quarter of 2020. Also in this case, the existence of a link captures a positive cross-border exposure $\alpha_{i j}$ between the two nodes $i, j$.

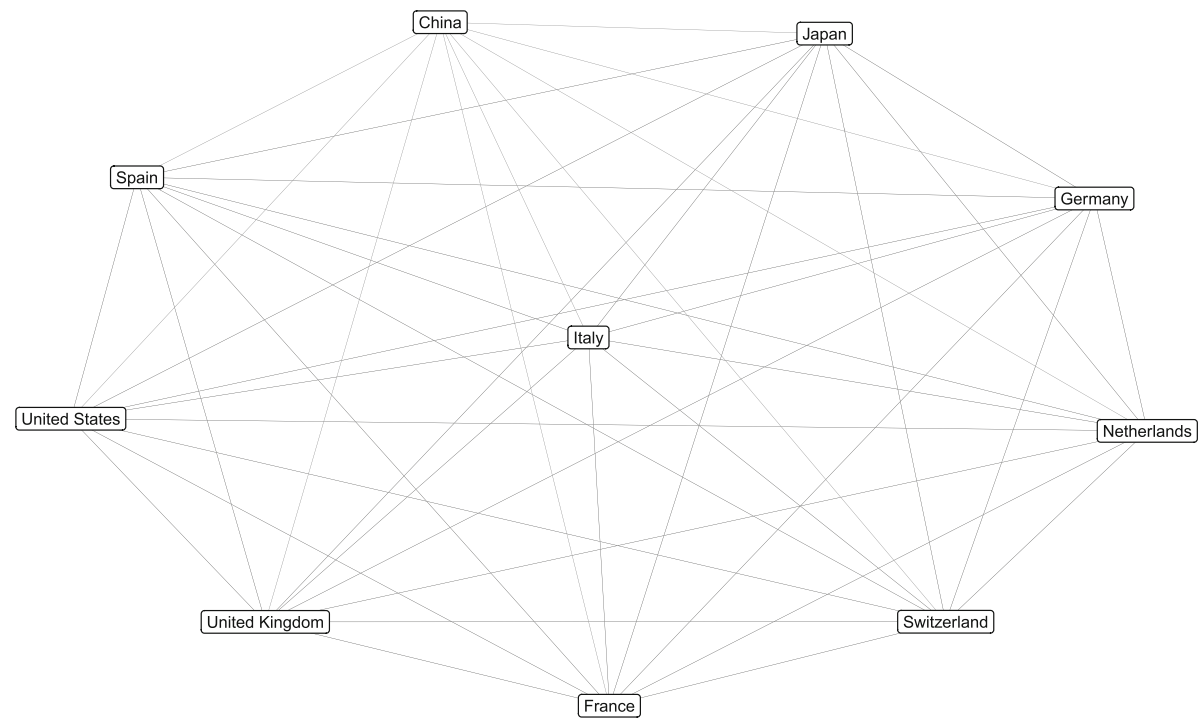

Fig. 4 Network of GS elements (i.e. countries where at least one global systemically important bank has its headquarter) for the LBS fourth quarter of 2020. Also in this case, the existence of a link captures a positive cross-border exposure $\alpha_{i j}$ between the two nodes $i, j$ 
Table 2 List of core countries in 2020

\begin{tabular}{|c|c|c|}
\hline Australia (AU) & Core & \\
\hline Austria (AT) & Core & \\
\hline Belgium (BE) & Core & \\
\hline Canada (CA) & Core & \\
\hline Chile (CL) & Core & \\
\hline China $(\mathrm{CN})$ & & GS \\
\hline Chinese Taipei (TW) & Core & \\
\hline Finland (FI) & Core & \\
\hline France (FR) & Core & GS \\
\hline Germany (DE) & Core & GS \\
\hline Greece (GR) & Core & \\
\hline Hong Kong SAR (HK) & Core & \\
\hline India (IN) & Core & \\
\hline Ireland (IE) & Core & \\
\hline Italy (IT) & Core & GS \\
\hline Japan (JP) & Core & GS \\
\hline South Korea (KR) & Core & \\
\hline Netherlands (NL) & Core & GS \\
\hline Norway (NO) & Core & \\
\hline Portugal (PT) & Core & \\
\hline Singapore (SG) & Core & \\
\hline Spain (ES) & Core & GS \\
\hline Sweden (SE) & Core & \\
\hline Switzerland $(\mathrm{CH})$ & Core & GS \\
\hline Turkey (TR) & Core & \\
\hline United Kingdom (GB) & Core & GS \\
\hline United States (US) & Core & GS \\
\hline
\end{tabular}

Some of them form also the group of GS countries, as indicated

This is due to the fact that the reporting countries make up a limited subset and some of the null values in the out-degree may be referred to the database incompleteness rather than to an absence of activity.

Finally, the out-degree shows remarkable fluctuations on the high values and data outlines the preferential exposure towards a limited number of banks/countries, with fluctuating exceptions.

\section{Results and discussion}

Herein we consider the analysis of the 64 BIS networks. They were built considering the CBS and LBS dataset fetched using the BIS API and cleaned from artifacts not regarding our purposes (e.g., rows containing summary data). The data processing, the network analysis and all simulations were conducted using the software $R$ (R Core Team 2014) with the igraph package (Csardi and Nepusz 2006). 
CBS

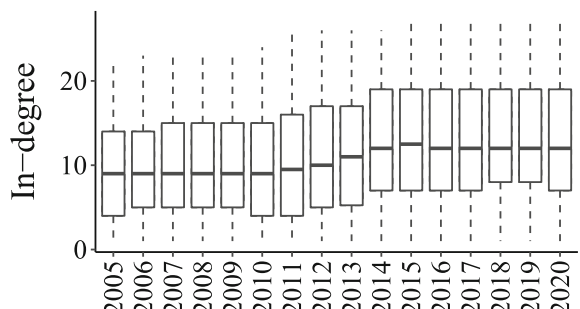

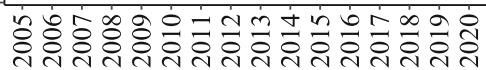

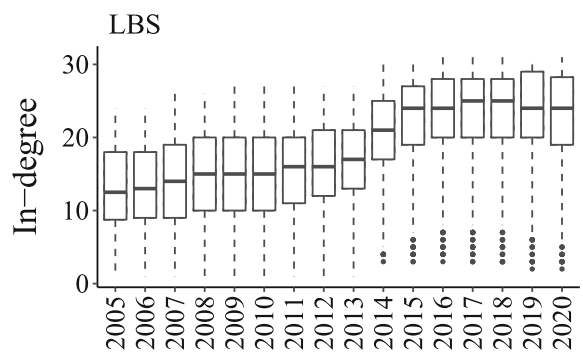

CBS

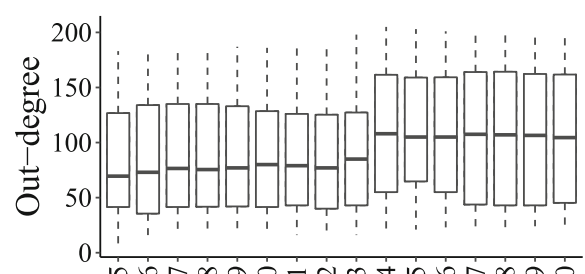

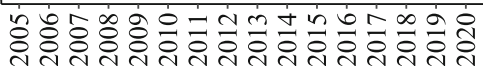

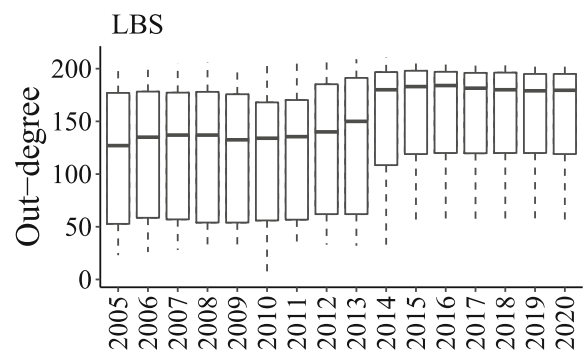

Fig. 5 Top panels: yearly distributions of in-degree (left) and out-degree (right) for CBS networks. Bottom panels: yearly distributions of in-degree (left) and out-degree (right) for LBS networks. In the out-degree distributions nodes with null out-degree values (peripheral elements) are filtered out

Equation (5) has been implemented through an algorithm which takes the network $N$, the values of $\xi$ and $\delta$ and of the components $\Gamma$ and $\Theta$ as inputs.

Some scenarios for the values of $\xi$ and $\delta$ and a peculiar setting for $\Gamma$ and $\Theta$ are considered, to include a large number of cases in the analysis of the resilience. Specifically, we take $\xi_{i}=\left(w_{\min } * 2^{10-i}\right)^{-1}$ with $w_{\min }$ as the lowest link weight over all the 64 instances and $i=1,2, \ldots, 10$; thus, the parameter space is: for CBS networks $\xi \in\{0.0021,0.0043,0.0087,0.0174,0.03498,0.0699,0.1399,0.2798,0.5597,1.1195\}$; for LBS $\xi \in\{0.0049,0.0098,0.0197,0.0394,0.0788,0.1576,0.3153,0.6307,1.2615$, $2.5231\}$; and $\delta \in\{0.1,0.25,0.5,0.75,1,2,5,10,20,50\}$. Summarising, we performed 100 computations for each of the CBS as well as for the LBS networks.

The highest values of $\xi$ has been chosen in order to be greater than the diameter $\bar{k}$. Needless to say, $\bar{k}$ is taken as the maximum diameter of the 64 considered networks.

Regarding the setting for $\Gamma$ and $\Theta$, we considered $\Gamma=\left\{\gamma_{i}=1, i=1, \ldots, \bar{k}\right\}$ and $\Theta=\left\{\theta_{i}=1 / \bar{k}\right.$, for $\left.i=1, \ldots, \bar{k}\right\}$. The former setting is associated to low propagation thresholds, so that a large number of shocks propagate. This condition can be seen as a prudential one, in that it avoids an a-priori mechanical removal of the effects of the shocks; the latter one considers uniform values of the $\theta$ 's, hence leading to a fair evaluation of all the terms contributing in the definition of the resilience measure $\mu_{(\Gamma, \xi)}(N)$.

Figures 6, 7 and 8 report the values of $\mu_{(\Gamma, \xi)}(N)$ for the CBS data, for every quarter and for each value of $\xi$, when considering all the values of $\delta$, namely $\delta \in$ $\{0.1,0.25,0.5,0.75,1,2,5,10,20,50\}$. According to the selected values of $\delta$, by merging the three figures we have ten panels.

As intuition suggests, we can generally say that the resilience of the networks decreases with respect to $\xi$. Indeed, by definition, a high value of the size $\xi$ leads to an easier propagation of the shock. 


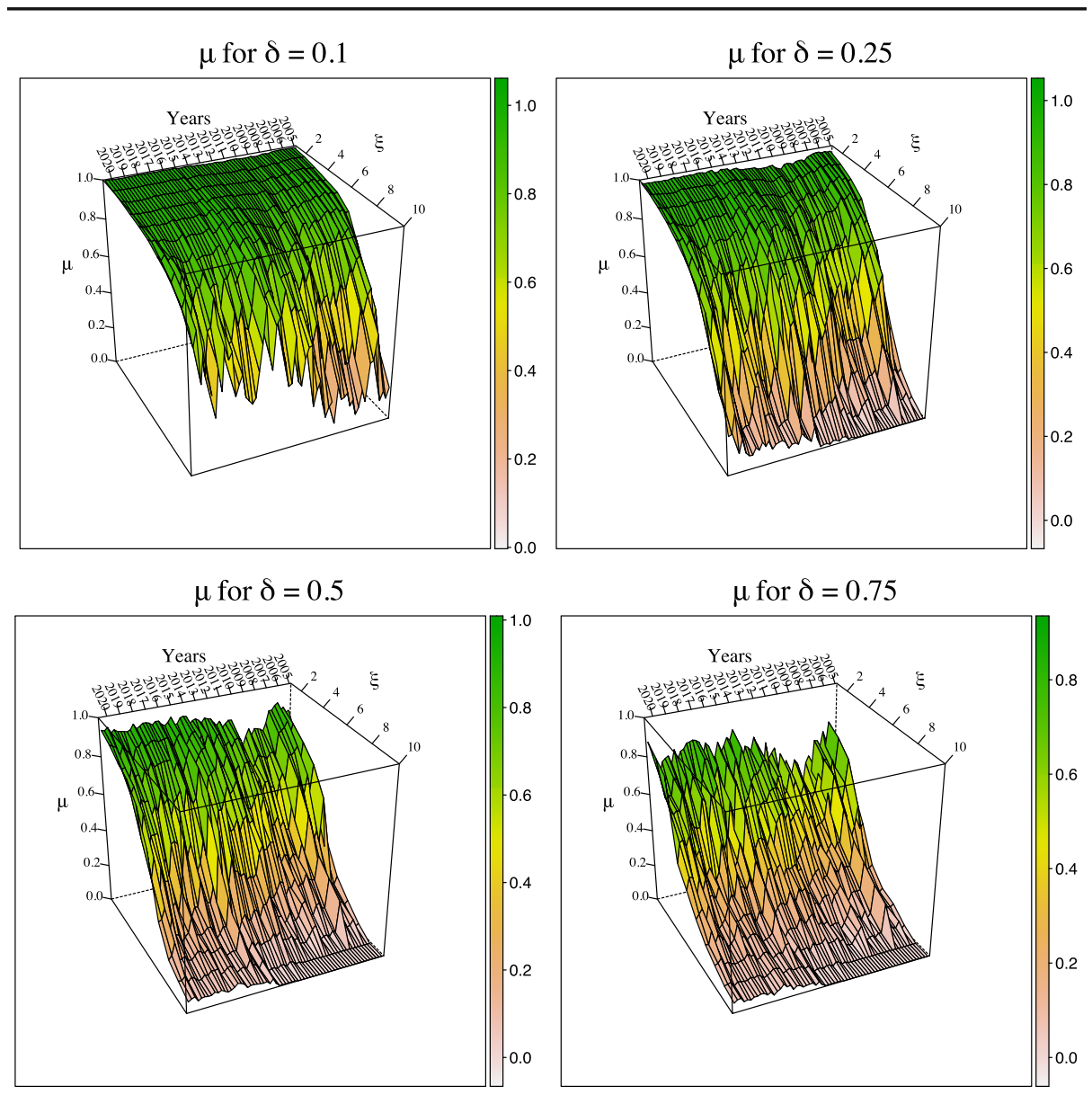

Fig. 6 Measures for the $\Gamma-\xi$-resilience $\mu_{(\Gamma, \xi)}(N)$ in the case of CBS networks for $\Gamma=\left\{\gamma_{i}=1, i=1, \ldots, \bar{k}\right\}$ and $\Theta=\left\{\theta_{i}=1 / \bar{k}\right.$, for $\left.i=1, \ldots, \bar{k}\right\}$. Different values of $\xi$ are reported on $x$-axis (as index of the vector), years on $y$-axis and values of $\mu_{(\Gamma, \xi)}(N)$ on $z$-axis. $(0.1 \leq \delta \leq 0.75)$

We also notice the existence of a critical threshold of $\xi$ above which we have the minimum level of resilience before year 2014 - the year following the Lehman Brothers failure and its consequences; this threshold reasonably decreases with respect to $\delta$, so that a strong reduction of the shocks propagation over the paths-i.e., small values of $\delta$-is associated to the propagation of shocks of large entity-i.e., shocks whose size is above the mentioned critical threshold.

Furthermore, the general level of the resilience measure decreases as $\delta$ increases. This outcome is due to the role of $\delta$ in the shock propagation process. Specifically, as $\delta$ increases, the propagation of the shock over a given path becomes more probable; thus, $\left|\mathcal{P}_{\Gamma, \xi}^{(k)} \cap \mathcal{P}_{\text {short }}\right|$ increases while $\left|\mathcal{P}^{(k)} \cap \mathcal{P}_{\text {short }}\right|$ remains constant, which implies that the resilience measure in (5) decreases.

In the extreme case of $\delta=0.1$-top-left panel in Fig. 6-the resilience attains values around the theoretical maximum for small values of $\xi$; moreover, the resilience surface does not show remarkable deviations across the years in this case. For high values of $\delta$-more 

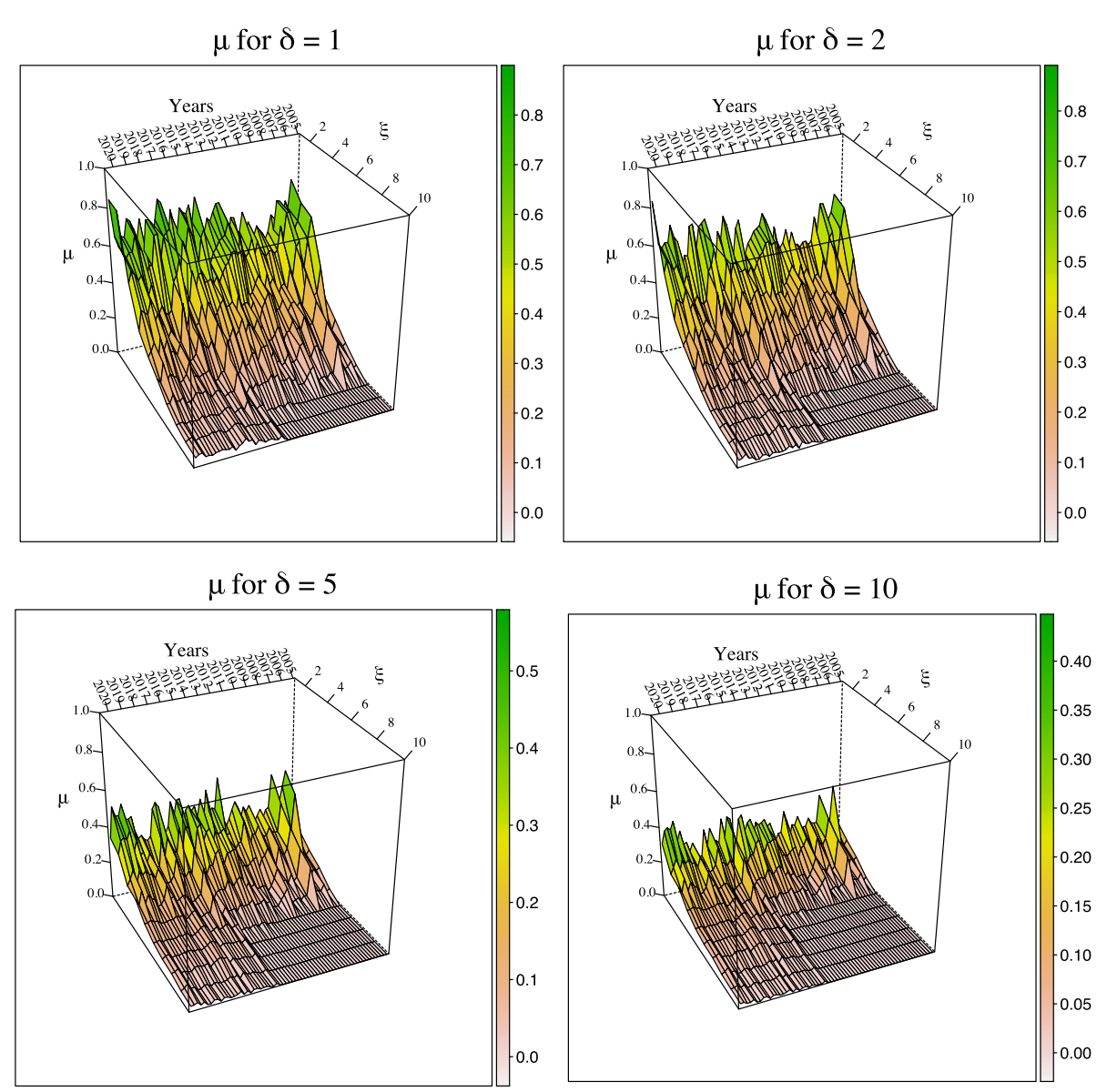

Fig. 7 Measures for the $\Gamma-\xi$-resilience $\mu_{(\Gamma, \xi)}(N)$ in the case of CBS networks for $\Gamma=\left\{\gamma_{i}=1, i=1, \ldots, \bar{k}\right\}$ and $\Theta=\left\{\theta_{i}=1 / \bar{k}\right.$, for $\left.i=1, \ldots, \bar{k}\right\}$. Different values of $\xi$ are reported on $x$-axis (as index of the vector), years on $y$-axis and values of $\mu_{(\Gamma, \xi)}(N)$ on $z$-axis. $(1 \leq \delta \leq 10)$

evidently in the bottom panels of Figs. 7 and 8-the resilience measure is close to zero for large values of $\xi$. This said, we observe a more scattered situation from 2017 to 2020, where one has traces of resilience-with resilience measure slightly greater than zero-also when $\xi=10$. Interestingly, there is a resilience peak in 2008 when $\delta>1$-i.e., when the shock is amplified as the distance from the shocked node increases. This resilience peak is associated to the year of the Lehman Brothers' failure-hence, to the relevant global financial crisis related to this catastrophic event. Such an outcome can be explained by the amplification of the cohesiveness of the network in correspondence of the financial distress; this is in agreement with the stylized fact that economic actors are more correlated in situations of economic turmoil.

For average values of $\delta$ - see the bottom panels of Figs. 6 and 7-we observe a collapse of the resilience measure in the triennium 2009-2012 - i.e., after the Lehman Brothers' failure. The relevance of such a collapse with respect to the adjacent years 2008 and 2013 is more evident for $0.5 \leq \delta \leq 5$. This is the period where the world experienced the consequences of 


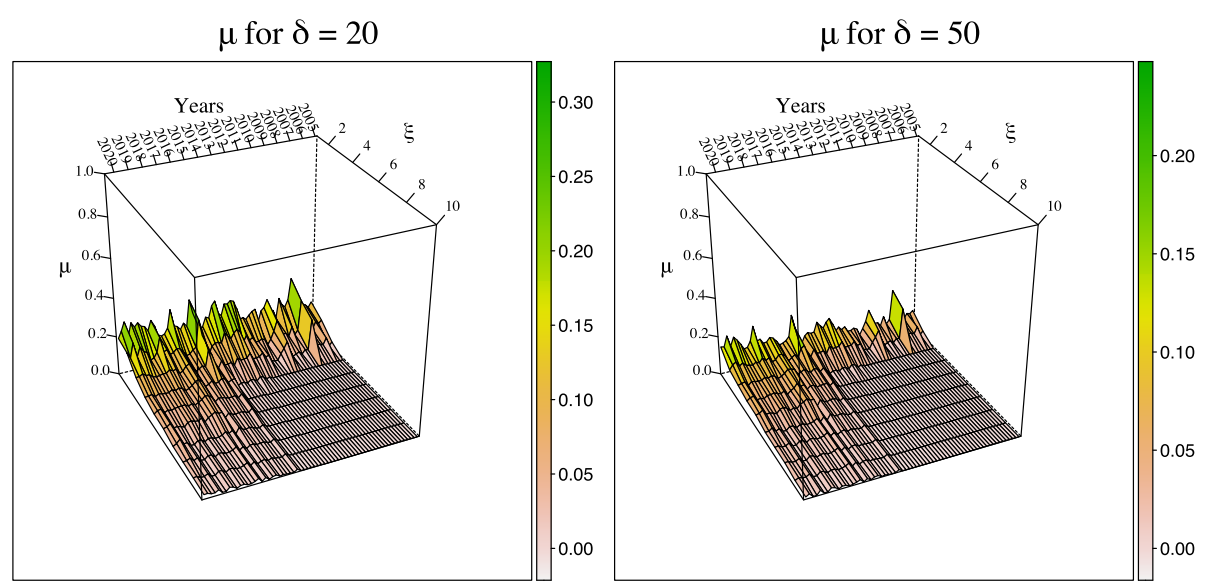

Fig. 8 Measures for the $\Gamma-\xi$-resilience $\mu_{(\Gamma, \xi)}(N)$ in the case of CBS networks for $\Gamma=\left\{\gamma_{i}=1, i=1, \ldots, \bar{k}\right\}$ and $\Theta=\left\{\theta_{i}=1 / \bar{k}\right.$, for $\left.i=1, \ldots, \bar{k}\right\}$. Different values of $\xi$ are reported on $x$-axis (as index of the vector), years on $y$-axis and values of $\mu_{(\Gamma, \xi)}(N)$ on $z$-axis. $(20 \leq \delta \leq 50)$

the financial turmoil of 2008, with a lower level of interbanking exposures among countries that is associated to a lower capability of the network to absorb shocks in a post-financial crisis.

The turbulence of the financial world registered from 2014 - with the Russian and Chinese market crashes and the bad performance of the global stock markets in 2018-is associated to the stronger interbanking relationships, hence leading to higher levels of the resilience measure after 2014. This argument is stressed in 2020-i.e., in the dramatic socio-financial crisis generated by the COVID-19 pandemic - where we have the highest level of resilience, also in accord to the case of 2008.

Furthermore, we observe that the resilience of the network decreases with the value of $\delta$. This outcome is in line with the role of the discount factor $\delta$ in the propagation of the shocks, and it represents a confirmation of the finding of our model. In this respect, the effect of $\delta$ on the resilience seems to be of remarkable relevance, mainly around the corner cases of $\delta=0$ and $\delta=1$. More interesting patterns are observed for fair values of $\delta$-i.e., for $\delta=0.4,0.5$, see Fig. 6. Indeed, in these cases, there is a collapse of the resilience around year 2014, after the period 2009-2013 of high resilience. The resilient period 2009-2013 corresponds to the one immediately following the Lehman Brothers' bankruptcy; hence, one can argue that the lowest level of interbanking exposures among countries is associated to a high capability of the network to absorb shocks in a post-financial crisis starting event period.

The results of the simulations of the LBS are reported in Figs. 9, 10, and 11. In general, we find a good agreement between the CBS and LBS across the parameter space, with the LBS networks slightly more resilient than the CBS ones and the flattening of the peaks in 2008 and 2020 in the LBS case. The robustness of the results is certified also by means of the Pearson's correlation between the time series of the indicator $\mu$ over CBS and LBS dataset for each value of $\delta$ and $\xi$-see Fig. 12 . 


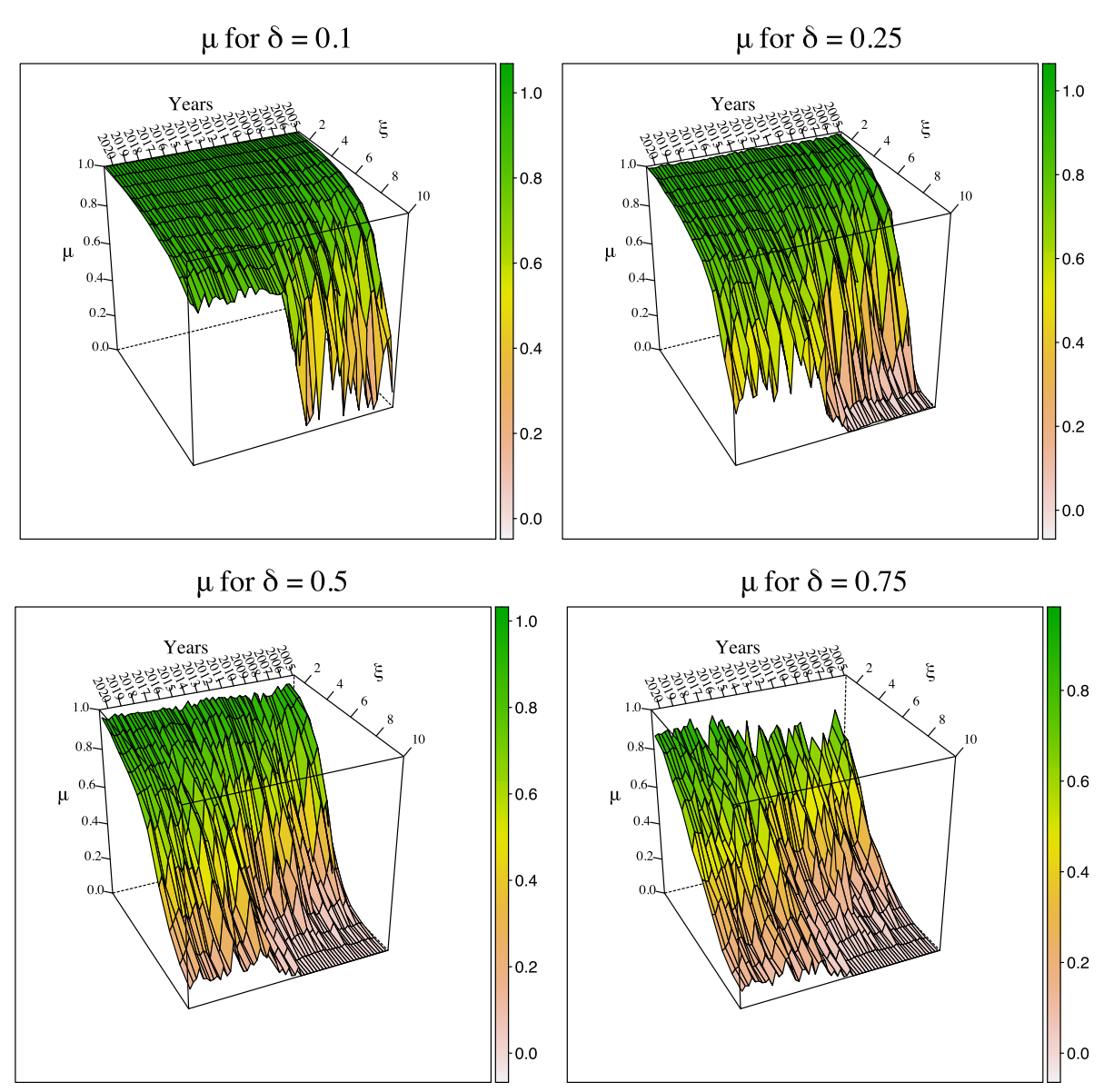

Fig. 9 Measures for the $\Gamma-\xi$-resilience $\mu_{(\Gamma, \xi)}(N)$ in the case of LBS networks for $\Gamma=\left\{\gamma_{i}=1, i=1, \ldots, \bar{k}\right\}$ and $\Theta=\left\{\theta_{i}=1 / \bar{k}\right.$, for $\left.i=1, \ldots, \bar{k}\right\}$. Different values of $\xi$ are reported on $x$-axis (as index of the vector), years on $y$-axis and values of $\mu_{(\Gamma, \xi)}(N)$ on $z$-axis. $(0.1 \leq \delta \leq 0.75)$

\section{Conclusions}

This paper proposes a novel resilience measure for networks that ranges in the unitary interval. Such a measure represents a relevant extension of the one proposed in Cerqueti et al. (2019). In particular, the computational complexity of the original measure has been removed by reasonably assuming — as in the cases of financial contagion — that shocks propagate over the shortest paths.

The introduced methodological device is tested over two meaningful networks built using the BIS database-with specific reference to the CBS data—of the interbank exposures gathered accordingly to the countries of the banks' headquarters. As robustness check, we also consider the networks built on the basis of the LBS data. The database is a precious source of information in that it provides evidence for the core-periphery structure of lending among banks worldwide, in line with previous results (see e.g. Cinelli et al. 2021 or Kojaku et al. 2018). The considered model provides insights on the evolution of network resilience 


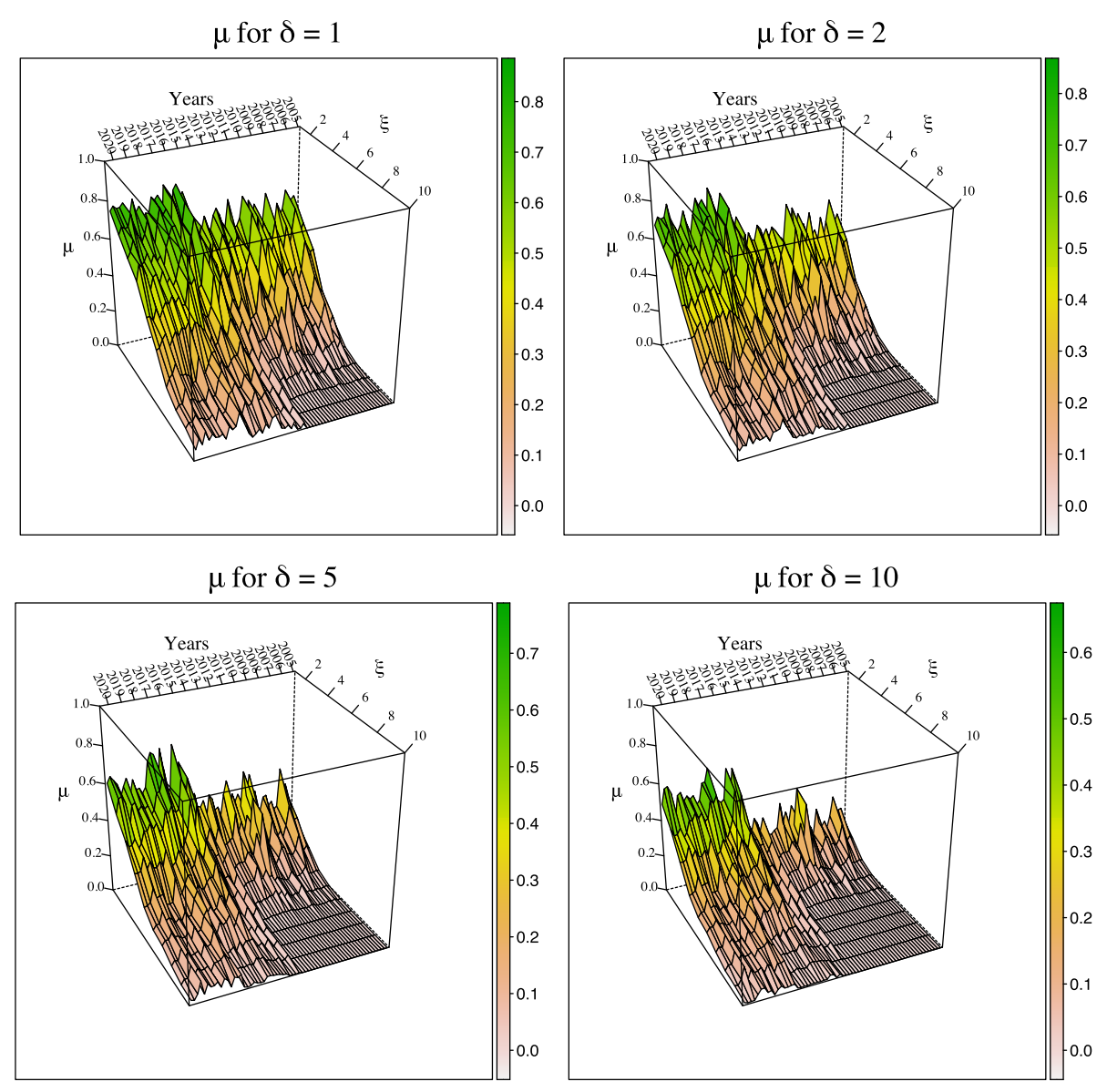

Fig. 10 Measures for the $\Gamma-\xi$-resilience $\mu_{(\Gamma, \xi)}(N)$ in the case of LBS networks for $\Gamma=\left\{\gamma_{i}=1, i=\right.$ $1, \ldots, \bar{k}\}$ and $\Theta=\left\{\theta_{i}=1 / \bar{k}\right.$, for $\left.i=1, \ldots, \bar{k}\right\}$. Different values of $\xi$ are reported on $x$-axis (as index of the vector), years on $y$-axis and values of $\mu_{(\Gamma, \xi)}(N)$ on $z$-axis. $(1 \leq \delta \leq 10)$.

in different time periods. Several scenarios for the empirical analysis have been presented and discussed.

The dependence of the resilience on the size of the shock and on the presence of a distancebased mitigating/amplifying term of the propagation has been also detected. In so doing, the employed resilience measure represents - at least in the treated cases-is a suitable instrument for assessing the stability of the financial system. Importantly, the empirical experiments offer a resilience measure of the BIS network which takes into full consideration some important financial crises of the 2000's, including the one started with the Lehman Brothers' failure in 2008 and the financial distress generated by the COVID-19 pandemic in 2020.

Results suggest that a high level of interbank exposures is able to immunize a system from the shocks in financial distress, while weakly interconnected banks generate a system of countries with a scarce attitude to absorb external shocks in presence of a financial turmoil. A possible interpretation of this outcome lies in the herd immunity against financial crisis of countries which are strongly linked through the banking system. Once such a link becomes 


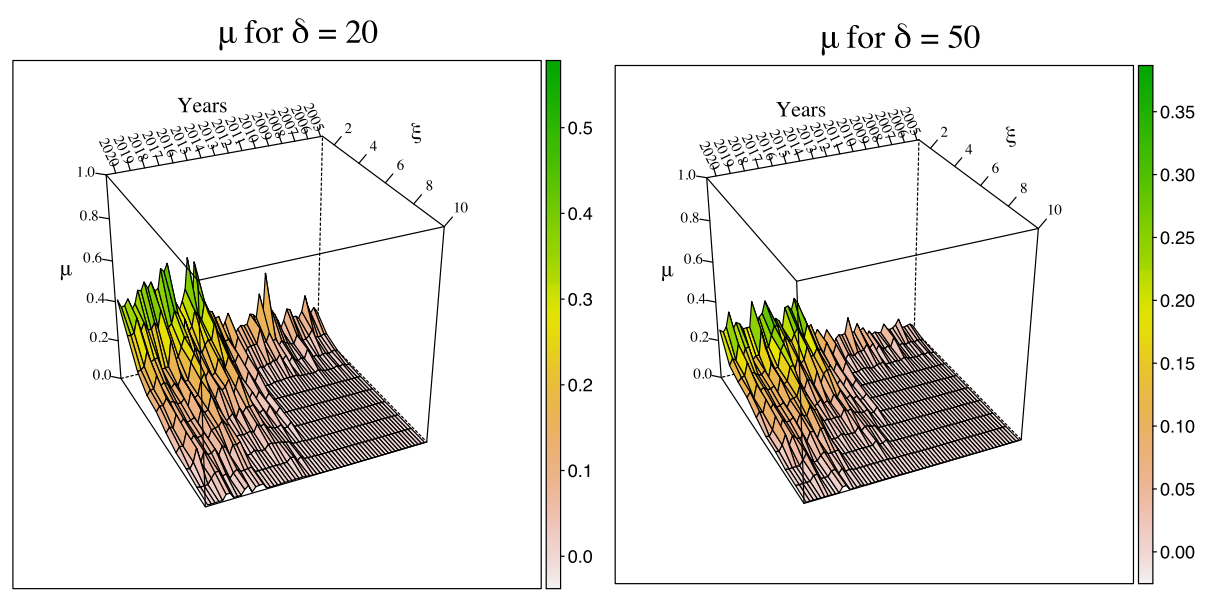

Fig. 11 Measures for the $\Gamma-\xi$-resilience $\mu_{(\Gamma, \xi)}(N)$ in the case of LBS networks for $\Gamma=\left\{\gamma_{i}=1, i=\right.$ $1, \ldots, \bar{k}\}$ and $\Theta=\left\{\theta_{i}=1 / \bar{k}\right.$, for $\left.i=1, \ldots, \bar{k}\right\}$. Different values of $\xi$ are reported on $x$-axis (as index of the vector), years on $y$-axis and values of $\mu_{(\Gamma, \xi)}(N)$ on $z$-axis. $(20 \leq \delta \leq 50)$.

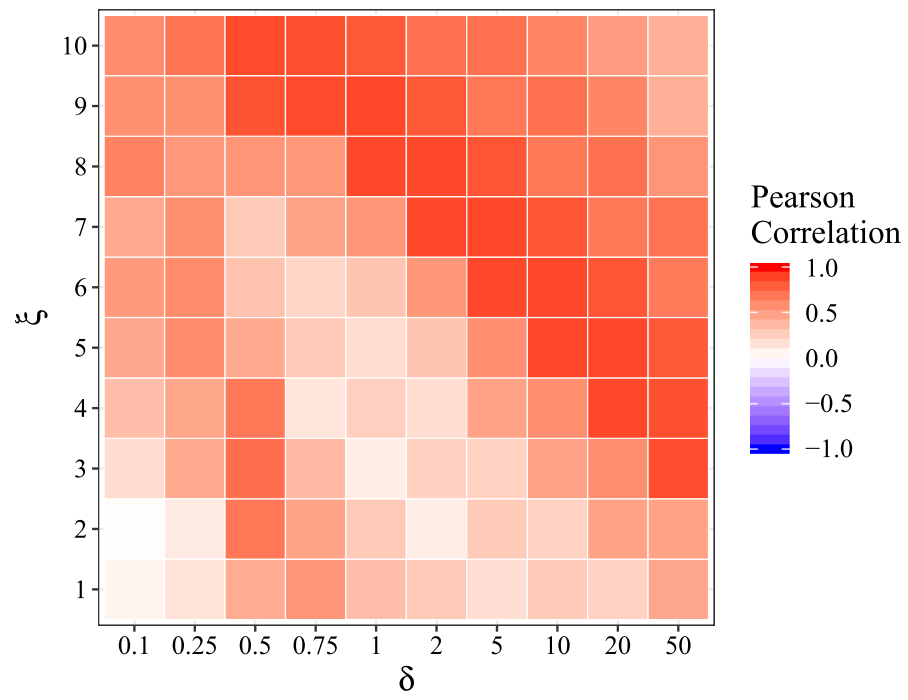

Fig. 12 Correlation matrix between the time series (ranging in each period from 2005 to 2020) of $\mu$ computed using CBS and LBS data across the parameter space. Since the parameter $\xi$ is a function of $w_{\min }$ we considered its rank values.

weak enough, then the situation is reverted and countries are particularly sensitive to the contagion as financial distress appears.

Under a purely methodological perspective, the measure herein introduced can be considered also as a general tool that can be applied to networks belonging to other financial contexts for measuring systemic risk. This aspect opens the field for future applications in other financial domains. The analysis performed with our method could be also used in order to identify the features of the shocks which are correlated with the resilience of the network, possibly suggesting countermeasures in order to build more resilient financial systems. 


\section{Appendix: BIS description}

BIS database is owned by 60 central banks, which cover about $95 \%$ of the world's GDP. The role of BIS is to provide a rigorous statistical description of international markets and financial intermediaries.

The available reporting countries — which are also those considered in the present studyare Australia, Austria, Belgium, Brazil, Canada, Chile, Chinese Taipei, Denmark, Finland, France, Germany, Greece, Hong Kong SAR, India, Ireland, Italy, Japan, Luxembourg, Mexico, Netherlands, Norway, Panama, Portugal, Singapore, South Korea, South Korea, Spain, Sweden, Switzerland, Turkey, United Kingdom, United States.

The counterpart countries are: Afghanistan, Albania, Algeria, Andorra, Angola, Argentina, Armenia, Aruba, Azerbaijan, Bahamas, Bahrain, Bangladesh, Barbados, Belarus, Belize, Benin, Bermuda, Bhutan, Bolivia, Bonaire, Sint Eustatius and Saba, Bosnia and Herzegovina, Botswana, Brunei, Bulgaria, Burkina Faso, Burundi, Cambodia, Cameroon, Cape Verde, Cayman Islands, Central African Republic, Chad, China, Colombia, Comoros, Congo, Congo Democratic Republic, Costa Rica, Cote d'Ivoire, Croatia, Cuba, Curacao, Cyprus, Czech Republic, Czechoslovakia, Djibouti, Dominica, Dominican Republic, Ecuador, Egypt, El Salvador, Equatorial Guinea, Eritrea, Estonia, Ethiopia, Faeroe Islands, Falkland Islands, Fiji, French Polynesia, Gabon, Gambia, Georgia, German Democratic Republic, Ghana, Gibraltar, Greenland, Grenada, Guatemala, Guernsey, Guinea, Guinea-Bissau, Guyana, Haiti, Honduras, Hungary, Iceland, Indonesia, Iran, Iraq, Isle of Man, Israel, Jamaica, Jersey, Jordan, Kazakhstan, Kenya, Kiribati, Kuwait, Kyrgyz Republic, Laos, Latvia, Lebanon, Lesotho, Liberia, Libya, Liechtenstein, Lithuania, Macao SAR, Macedonia (FYR), Madagascar, Malawi, Malaysia, Maldives, Mali, Malta, Marshall Islands, Mauritania, Mauritius, Micronesia, Moldova, Mongolia, Montenegro Morocco, Mozambique, Myanmar, Nauru, Nepal, Netherlands Antilles, New Caledonia, New Zealand, Nicaragua, Nigeria, North Korea, Oman, Pakistan, Palau, Palestinian Territory, Papua New Guinea, Paraguay, Peru, Philippines, Poland, Qatar, Romania, Russia Rwanda, Samoa, San Marino, Sao Tome and Principe, Saudi Arabia, Senegal, Serbia, Serbia and Montenegro, Seychelles, Sierra Leone, Sint Maarten, Slovakia, Slovenia, Solomon Islands, Somalia, South Africa, South Sudan, Soviet Union, Sri Lanka, St. Helena and Dependencies, St. Lucia, St. Vincent and the Grenadines, Sudan, Suriname, Swaziland, Syria, Tajikistan, Tanzania, Thailand, Timor Leste, Togo, Tonga, Trinidad and Tobago, Tunisia, Turkmenistan, Turks and Caicos Islands, Tuvalu, Uganda, Ukraine, United Arab Emirates, Uruguay, US Pacific Islands, Uzbekistan, Vanuatu, Vatican City State, Venezuela, Vietnam, Wallis and Futuna, Yemen, Yugoslavia, Zambia, Zimbabwe.

Countries belong to three different groups (seeBongini et al. 2018): 26 core countries, which report to the BIS both outgoing and incoming exposures to the BIS; 10 Global Systemically (GS) countries, each of them being the headquarter of at least one Global Systemically Important Banks (G-SIB). Such banks have to obey more stringent requirements in reporting their data (see http://www.fsb.org/2017/11/fsb-publishes-2017-g-sib-list/; data on core and GS have been included also in Table 2); the peripheral countries, for which only the inflows are available.

\section{References}

Acemoglu, D., Ozdaglar, A., \& Tahbaz-Salehi, A. (2015). Systemic risk and stability in financial networks. American Economic Review, 105(2), 564608. 
Albert, R., Jeong, H., \& Barabási, A.-L. (2000). Error and attack tolerance of complex networks. Nature, 406, 378-382.

Allen, F., \& Babus, A. (2008) Networks in finance. Wharton Financial Institutions CenterWorking Paper No. 08-07.

Ahuja, R. K., Magnanti, T. L.,\& Orlin, J. B.(1988) Network flows: Theory, Algorithms, and Applications, Pearson College Div.

Bongini, P., Clemente, G. P., \& Grassi, R. (2018). Interconnectedness, G-SIBs and network dynamics of global banking. Finance Research Letters, 27, 185-192.

Cerqueti, R., Clemente, G. P., \& Grassi, R. (2020). Systemic risk assessment through high order clustering coefficient. Annals of Operations Research. https://doi.org/10.1007/s10479-020-03525-8

Cerqueti, R., Ferraro, G., \& Iovanella, A. (2019). Measuring network resilience through connection patterns. Reliability Engineering and System Safety, 188, 320-329.

Chen, P. Y., \& Cheng, S. M. (2015). Sequential defence against random and intentional attacks in complex networks. Physical Review E, 91, 022805.

Chevallier, J., Goutte, S., Guerreiro, D., Saglio, S., \& Sanhaji, B. (Eds.). (2019). International financial markets (Vol. 1). Routledge.

Cinelli, M., Ferraro, G., \& Iovanella, A. (2017). Resilience of core-periphery networks in the case of rich-club. Complexity, 2017, 6548362.

Cinelli, M., Ferraro, G., Iovanella, A., \& Rotundo, G. (2021). Assessing the impact of incomplete information on the resilience of financial networks. Annals of Operations Research, 299, 721-745.

Csardi G,\& Nepusz T. (2006). The igraph software package for complex network research. InterJournal Complex System, 1695. http://igraph.org.

D’Agostino, G., Scala, A., Zlatic, V., \& Caldarelli, G. (2012). Robustness and assortativity for diffusion-like processes in scale-free networks. Europhysics Letters, 97(6), 68006.

Demetrescu, C., Goldberg, A. V.,\& Johnson, D. S. (Eds.), (2009). The shortest path problem: Ninth DIMACS implementation challenge (Vol. 74). American Mathematical Soc.

Demir, M., \& Onder, Z. (2019). Financial connectivity and excessive liquidity: Benefit or risk? Journal of International Financial Markets, Institutions and Money, 62, 203-221.

Dumontaux, N., \& Pop, A. (2013). Understanding the market reaction to shockwaves: Evidence from the failure of Lehman Brothers. Journal of Financial Stability, 9(3), 269-286.

Elliott, M., Golub, B., \& Jackson, M. O. (2014). Financial networks and contagion. American Economic Review, 104(10), 3115-53.

Ferrraro, G., \& Iovanella, A. (2018). Clairvoyant targeted attack on complex networks. International Journal of Computational Economics and Econometrics, 8(1), 41-62.

Fortune, S., Hopcroft, J. E., \& Wyllie, J. (1980). The directed subgraph homeomorphism problem. Theoretical Computer Science, 10, 111-21.

Gao, J., Barzel, B., \& Barabási, A.-L. (2016). Universal resilience patterns in complex networks. Nature, 530(7590), 307-312.

Giudici, P., \& Spelta, A. (2016). Graphical network models for international financial flows. Journal of Business and Economic Statistics, 34(1), 128-138.

Glasserman, P., \& Peyton Young, H. (2016). Contagion in financial networks. Journal of Economic Literature, 54(3), 779-831.

Kojaku, S., Cimini, G., Caldarelli, G., \& Masuda, N. (2018). Structural changes in the interbank market across the financial crisis from multiple core-periphery analysis. Journal of Network Theory in Finance, 4(3), $33-51$.

Iyer, S., Killingback, T., Sundaram, B., \& Wang, Z. (2013). Attack robustness and centrality of complex networks. PLoS ONE, 8(4), e59613.

Minoiu, C., Kang, C., Subrahmanian, V., \& Berea, A. (2015). Does financial connectedness predict crises? Quantitative Finance, 15, 607-624.

Minoiu, C., \& Reyes, J. A. (2013). A network analysis of global banking: 1978-2010. Journal of Financial Stability, 9(2), 168-184.

Nagurney, A., \& Ke, K. (2006). Financial networks with intermediation: Risk management with variable weights. European Journal of Operation Research, 172, 40-63.

Nedovic, L., \& Devedzic, V. (2002). Expert systems in finance-A cross-section of the field. Expert Systems with Applications, 23(1), 49-66.

Peron, T. K., da Fontoura Costa, L., \& Rodrigues, F. A. (2012). The structure and resilience of financial market networks. Chaos, 22, 013117.

Rada, R. (2008). Expert systems and evolutionary computing for financial investing: A review. Expert Systems with Applications, 34(4), 2232-2240. 
R Core Team. R: A language and environment for statistical computing. $R$ Foundation for Statistical Computing, Vienna, Austria 2014. http://www.R-project.org.

Rosas-Casals, M., Bologna, S., Bompard, E. F., D’Agostino, G., Ellens, W., Pagani, G. A., \& Verma, T. (2015). Knowing power grids and understanding complexity science. International Journal of Critical Infrastructures, 11(1), 4-14.

Scala, A., Lucentini, P. G. D. S., Caldarelli, G., \& D’Agostino, G. (2016). Cascades in interdependent flow networks. Physica D: Nonlinear Phenomena, 323, 35-39.

Tonzer, L. (2015). Cross-border interbank networks, banking risk and contagion. Journal of Financial Stability, $18,19-32$.

Publisher's Note Springer Nature remains neutral with regard to jurisdictional claims in published maps and institutional affiliations. 